\title{
THE CAUCHY DATA AND THE SCATTERING RELATION*
}

\author{
GUNTHER UHLMANN ${ }^{\dagger}$
}

1. Introduction. As mentioned in the preface to this volume a combination of unique continuation results with the boundary control method has led to the solution of the inverse problem of determining a metric of a Riemannian manifold (with boundary) from the dynamic Dirichlet-toNeumann map associated with the wave equation. Although these results are very satisfactory it requires too much information. By just looking at the singularities of the dynamic Dirichlet-to-Neumann (DN) map one can determine the boundary distance function (the minimal travel time along geodesics connecting points on the boundary of a Riemannian manifold) in the case that there are no conjugate points of the metric, i.e. no caustics. This is shown in Section 3 of this article using geometrical optics expansions. A natural question to ask if one can determine the metric from this data alone; this question is at the center of the boundary rigidity problem studied in Riemannian geometry which is one of the main topics of this volume.

Microlocal analysis is also used in Section 4 of this paper to show that from the DN map for the wave equation one can determine the scattering relation (or lens map). This result does not assume that there are no caustics. Roughly speaking this encodes the information of all travel times not just the ones given by minimizing geodesics. An approach to solve the inverse problem of finding the Riemannian metric from the scattering relation proposed by Stefanov and the author is described in detail.

In Sections 5 and 6 of this paper we consider elliptic inverse boundary value problems. We study the problem of determining an anisotropic or isotropic conductivity from the corresponding DN map or set of Cauchy data which encodes all the electrical measurements that can be made at the boundary. We also survey a result of Bukhgeim and the author showing that if one measures the set of Cauchy data for isotropic conductivites on particular sets of the boundary then one can determine uniquely the conductivity. The method of proof uses new Carleman estimates.

The author believes that there is a connection between the scattering relation and the set of Cauchy data that remains to be unraveled. This explains the title of this article.

2. The hyperbolic Dirichlet-to-Neumann map. Let $\Omega$ denote a bounded domain on $\mathbf{R}^{n}, n \geq 2$ and let $g$ be a Riemannian metric on $\Omega$. In local coordinates $g$ is represented by $g(x)=\left(g_{i j}(x)\right)$ a positive definite,

*The research for this paper was partly supported by NSF and a John Simon Guggenheim fellowship.

${ }^{\dagger}$ Department of Mathematics, University of Washington, Seattle, WA 98195-4350. 
symmetric matrix on $\bar{\Omega}$. We assume for simplicity that the Riemannian metric $g$ is smooth (several of the results are valid assuming finite smoothness). The Euclidean metric is denoted by $e=\left(\delta_{i j}\right)$.

We denote by $\Delta_{g}$ the Laplace-Beltrami operator associated to the metric $g$, i.e. in local coordinates

$$
\Delta_{g}=(\operatorname{det} g)^{-\frac{1}{2}} \sum_{i, j=1}^{n} \frac{\partial}{\partial x_{i}}(\operatorname{det} g)^{\frac{1}{2}} g^{i j} \frac{\partial}{\partial x_{j}}
$$

where $\left(g^{i j}\right)=\left(g_{i j}\right)^{-1}, \operatorname{det} g=\operatorname{det}\left(g_{i j}\right)$.

We consider the solution of the initial-boundary value problem for the wave equation associated to the Laplace-Beltrami operator:

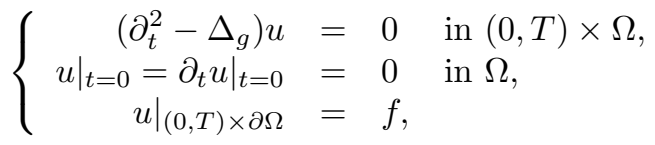

where $f \in H_{\text {loc }}^{2}, f=0$ for $t<0$. Denote by $\nu=\nu(x)$ the outer normal to $\partial \Omega$ at $x \in \partial \Omega$. We define the hyperbolic (dynamic) Dirichlet-to-Neumann (HDN) map $\Lambda_{g}^{h}$ by

$$
\Lambda_{g}^{h} f:=\left.(\operatorname{det} g)^{\frac{1}{2}} \sum_{i, j=1}^{3} \nu_{i} g^{i j} \frac{\partial u}{\partial x_{j}}\right|_{(0, T) \times \partial \Omega} .
$$

The inverse problem is whether knowledge of the HDN map determines the metric $g$ uniquely. However, any metric isometric to $g$ gives rise to the same boundary measurements. More precisely let

$$
\psi: \bar{\Omega} \longrightarrow \bar{\Omega},\left.\quad \psi\right|_{\partial \Omega}=\text { Identity }
$$

be a diffeomorphism. Then

$$
\Lambda_{g}^{h}=\Lambda_{\psi^{*} g}^{h},
$$

where $\psi^{*} g=\left(D \psi \circ g \circ{ }^{t} D \psi\right) \circ \psi^{-1}$ denotes the pull back of the metric under the diffeomorphism $\psi$.

This is in fact the only obstruction to unique identifiability of the metric from the HDN map. This result is a consequence of the paper [6], which uses the boundary control method (BC) pioneered by Belishev (see [5] for a survey and [18] for a recent book). The article [19] in this volume has further developments on the boundary control method and applications to inverse problems. In turn the $\mathrm{BC}$ method depends on a Holmgren type uniqueness theorem for hyperbolic equations. This breakthrough result was proven by Tataru [43] (see also [33]), after the BC has been formulated, using very novel Carleman type estimates. For more details see the article by Tataru [44] in these proceedings. 
The precise statement of the uniqueness result is:

THEOREM 2.1. Let $g_{k} \in C^{\infty}(\bar{\Omega})$, be two Riemannian metrics on $\bar{\Omega}$ and denote by $\Lambda_{g_{k}}^{h}, k=1,2$ the corresponding HDN maps. Assume

$$
\Lambda_{g_{1}}^{h}=\Lambda_{g_{2}}^{h}
$$

then there exists, for $T$ sufficiently large, a diffeomorphism $\psi: \bar{\Omega} \rightarrow \bar{\Omega}$ with $\left.\psi\right|_{\partial \Omega}=I d$, such that $\psi^{*} g_{1}=g_{2}$.

The result of [6] and [43] is valid for general compact Riemannian manifolds with boundary. Some stability estimates are known in this problem but these are in weak topology [20]. Hölder type stability estimates for the case that the metrics are close to the Euclidean one were proven in [35]. It is an interesting open problem to find sharp stability estimates for the inverse problem of determining a Riemannian metric from the hyperbolic DN map.

3. The boundary distance function. The problem considered in the previous section is highly overdetermined in dimensions $n \geq 2$. The Schwartz kernel of the HDN map depends on $2 n-1$ variables and the metric $g$ depends on $n$ variables.

We will show in this section that if $(\Omega, g)$ is simple that by looking at the singularities of the HDN we can determine the boundary distance function $d_{g}(x, y), x, y \in \partial \Omega$, that is, the travel times of geodesics going through the domain. The boundary distance function is a function of $2 n-2$ variables. Thus the inverse problem of determining the Riemannian metric from the boundary distance function is formally determined in two dimensions and formally overdetermined in dimensions $n \geq 3$. Some recent results on this problem are reviewed in this volume [10]. The linearized problem consists of determining a tensor field from its integral along geodesics. Sharafutdinov surveys recent developments on this problem in the article [34] in this volume.

Under the assumptions of Theorem 2.1 we can assume that, after a change of variables which is the identity at the boundary, the two metrics $g_{1}$ and $g_{2}$ have the same Taylor series at the boundary [39]. Therefore we can extend both metrics smoothly to be equal outside outside $\Omega$ and Euclidean outside a ball of radius $R$. We denote the extensions to $\mathbf{R}^{n} g_{j}, j=1,2$, as before. Let $u_{j}(t, x, \omega)$, be the solution of the continuation problem

$$
\left\{\begin{aligned}
\frac{\partial^{2} u}{\partial t^{2}}-\Delta_{g_{j}} u_{j} & =0, \text { in } \mathbb{R}^{n} \times \mathbb{R} \\
u_{j}(t, x) & =\delta(t-x \cdot \omega), t<-R
\end{aligned}\right.
$$

where $\omega \in \mathbb{S}^{n-1}=\left\{x \in \mathbf{R}^{n} ;|x|=1\right\}$.

It was shown in [39] that under the hypotheses of Theorem 2.1 the two solutions coincide outside $\Omega$, namely

$$
u_{1}(t, x, \omega)=u_{2}(t, x, \omega), x \in \mathbb{R}^{n} \backslash \Omega .
$$


In the case that the manifold $\left(\Omega, g_{j}\right), j=1,2$ is simple, we will use methods of geometrical optics to construct solutions of (4) to show if the HDN map of $g_{1}$ and $g_{2}$ are the same then the boundary distance functions of the metrics $g_{1}$ and $g_{2}$ coincide.

3.1. Real-geometrical optics. Let $g$ denotes a smooth Riemannian metric which is Euclidean outside a ball of radius $R$.

We will construct solutions to the continuation problem for the metric $g$ (which is either $g_{1}$ or $g_{2}$ ). We fix $\omega$. Let us assume that there is a solution to Equation (4) of the form

(6) $u(t, x, \omega)=a(x, \omega) \delta(t-\phi(x, \omega))+v(t, x, \omega), \quad u=0, t<-R$,

where $a, \phi$ are functions to be determined and $v \in L_{\text {loc }}^{2}$ Notice that in order to satisfy the initial conditions in (4), we require that

$$
a=1, \quad \phi(x, \omega)=x \cdot \omega \quad \text { for } x \cdot \omega<-R .
$$

By replacing Equation (6) in Equation (4), it follows that

$$
\begin{aligned}
\frac{\partial^{2} u}{\partial t^{2}}-\Delta_{g} u= & A \delta^{\prime \prime}(t-\phi(x, \omega))+B \delta^{\prime}(t-\phi(x, \omega)) \\
& -\left(\Delta_{g} a\right) \delta(t-\phi(x, \omega))+\frac{\partial^{2} v}{\partial t^{2}}-\Delta_{g} v,
\end{aligned}
$$

where

$$
\begin{aligned}
& A=a(x, \omega)\left(1-\sum_{i, j=1}^{n} g^{i j} \frac{\partial \phi}{\partial x^{i}} \frac{\partial \phi}{\partial x^{j}}\right) \\
& B=2 \sum_{j, k=1}^{n} g^{j k} \frac{\partial a}{\partial x^{k}} \frac{\partial \phi}{\partial x^{j}}+a \Delta_{g} \phi .
\end{aligned}
$$

We choose the functions $\phi, a$ in the expansion (6) to eliminate the singularities $\delta^{\prime \prime}$ and $\delta^{\prime}$ and then construct $v$ so that

$$
\frac{\partial^{2} v}{\partial t^{2}}-\Delta_{g} v=\left(\Delta_{g} a\right) \delta(t-\phi(x, \omega)), \quad v=0, t<-R .
$$

3.1.1. The Eikonal equation. In order to solve the equation $A=0$, it is sufficient to solve the equation

$$
\sum_{i, j=1}^{n} g^{i j} \frac{\partial \phi}{\partial x^{i}} \frac{\partial \phi}{\partial x^{j}}=1, \quad \phi(x, \omega)=x \cdot \omega, \quad x \cdot \omega<-R .
$$

Equation (12) is known as the Eikonal Equation. Here we will describe a method, using symplectic geometry, to solve this equation. 
Let $H_{g}(x, \xi)=\frac{1}{2}\left(\sum_{i, j=1}^{n} g^{i j}(x) \xi_{i} \xi_{j}-1\right)$ the Hamiltonian associated to the metric $g$. Note that the metric induced by $g$ in the cotangent space $T^{*} \mathbb{R}^{n}$ is given by the principal symbol of the Laplace-Beltrami operator $g^{-1}(x, \xi)=\sum_{i, j=1}^{n} g^{i j}(x) \xi_{i} \xi_{j}$. The equation (12) together with the initial condition can be rewritten as

$$
H_{g}(x, d \phi)=0, \quad \phi(x, \omega)=x \cdot \omega, x \cdot \omega<-R
$$

where $d \phi=\sum_{i=1}^{n} \frac{\partial \phi}{\partial x^{i}} d x^{i}$ is the differential of $\phi$.

Let $S=\left\{(x, \xi): H_{g}(x, \xi)=0\right\}$, and let $M_{\phi}=\left\{(x, \nabla \phi(x)): x \in \mathbb{R}^{n}\right\}$, then solving Equation (12), is equivalent to finding $\phi$ such that

$$
M_{\phi} \subset S \text {, with } M_{\phi}=\{(x, \omega) ; x \cdot \omega<-R\} .
$$

In order to find $\phi$ so that (13) is valid we need to find a Lagrangian submanifold $L$ so that $L \subset S, L=\{(x, \omega) ; x \cdot \omega<-R\}$ and the projection on $L$ of $T^{*} \mathbb{R}^{n}$ to $\mathbb{R}^{n}$ is a diffeomorphism [11]. We will construct such a Lagrangian manifold by flowing out from $N=\{(x, \omega): x \cdot \omega=s$ and $s<-R\}$ by the geodesic flow associated to the metric $g$. We recall the definition of geodesic flow.

We define the Hamiltonian vector field associated to $H_{g}$

$$
V_{g}=\left(\frac{\partial H_{g}}{\partial \xi},-\frac{\partial H_{g}}{\partial x}\right)
$$

The bicharacteristics are the integral curves of $H_{g}$

$$
\frac{d}{d s} x_{m}=\sum_{j=1}^{n} g^{m j} \xi_{j}, \quad \frac{d}{d s} \xi_{m}=-\frac{1}{2} \sum_{i, j=1}^{n} \frac{\partial g^{i j}}{\partial x_{m}} \xi_{i} \xi_{j}, \quad m=1, \ldots, n .
$$

The projections of the bicharacteristics contained in $H_{g}(x, \xi)=0$ in the $x$ variable are the geodesics of the metric $g$ and the parameter $s$ denotes arc length. We denote the associated geodesic flow by

$$
X_{g}(s)=\left(x_{g}(s), \xi_{g}(s)\right) .
$$

If we impose the condition that the bicharacteristics are in $S$ initially, then they belong to $S$ for all time, since the Hamiltonian vector field $V_{g}$ is tangent to $S$. The Hamiltonian vector field is transverse to $N$ then the resulting manifold obtained by flowing $N$ along the integral curves of $V_{g}$ will be a Lagrangian manifold $L$ contained in $S$. We shall write $L=X_{g}(N)$.

Now the projection of $N$ into the base space is a diffeomorphism so that $L=\left\{\left(x, d_{x} \phi\right)\right\}$ locally near a point of $N$. We can construct a global solution of (13) near $\Omega$ if the manifold is simple. We recall:

DEFINITION 3.1. Let $\Omega$ be a bounded domain of Euclidean space with smooth boundary and $g$ a Riemannian metric on $\bar{\Omega}$. We say that $(\Omega, g)$ 
is simple if given two points on the boundary there is a unique minimizing geodesic joining the two points on the boundary and, moreover, $\partial \Omega$ is geodesically convex.

If $(\Omega, g)$ is simple then we extend the metric smoothly in a small neighborhood so that the metric $g$ is Euclidean in a small neighborhood of $\Omega$ so that the metric is still simple. In this case we can solve the Eikonal equation globally in a neighborhood of $\Omega$.

3.1.2. The transport equation. The equation $B=0$ is equivalent to solving the following equation:

$$
\sum_{i, j=1}^{n} g^{i j} \frac{\partial \phi}{\partial x^{j}} \frac{\partial a}{\partial x^{i}}+\frac{a}{2} \Delta_{g} \phi=0 .
$$

Equation 16 is called the transport equation. It is a vector field equation for $a(x, \omega)$, which is solved by integrating along the integral curves of the vector field $v=\sum_{i, j=1}^{n} g^{i j} \frac{\partial \phi}{\partial x^{j}} \frac{\partial}{\partial x^{2}}$. It is an easy computation to prove that $v$ has length 1 and that the integral curves of $v$ are the geodesics of the metric $g$.

The solution of the transport equation (16) is then given by:

$$
a(x, \omega)=\exp \left(-\frac{1}{2} \int_{\gamma} \Delta_{g} \phi\right),
$$

where $\gamma$ is the unique geodesic such that $\gamma(0)=y, \dot{\gamma}(0)=\omega, y \cdot \omega=-R$ and $\gamma$ passes through $x$. If $(\Omega, g)$ is a simple manifold then $a \in C^{\infty}\left(\mathbb{R}^{n}\right)$.

To end the construction of the real-geometrical optics solutions we observe that the function $v(t, x, \omega) \in L_{\text {loc }}^{2}$ by using standard regularity results for hyperbolic equations.

Now we state the main result of this section:

TheOREM 3.1. Let $\left(\Omega, g_{i}\right), i=1,2$ be simple metrics. Assume that $\Lambda_{g_{1}}^{h}=\Lambda_{g_{2}}^{h}$. Then $d_{g_{1}}=d_{g_{2}}$.

Sketch of proof. Assume that we have two metrics $g_{1}, g_{2}$ with the same HDN map. Then by (5) the solutions of (4) are the same outside $\Omega$. Therefore the main singularity of the solutions in the geometrical optics expansion must be the same outside $\Omega$. Thus we conclude that

$$
\phi_{1}(x, \omega)=\phi_{2}(x, \omega), \quad x \in \mathbf{R}^{n} \backslash \Omega .
$$

Now $\phi_{j}(x, \omega)$ measures the geodesic distance to the hyperplane $x \cdot \omega=$ $-R$ in the metric $g$. From this we can easily conclude that the geodesic distance between two points in the boundary for the two metrics is the same, that is $d_{g_{1}}(x, y)=d_{g_{2}}(x, y), x, y \in \partial \Omega$.

This type of argument was used in [31] to study a similar inverse problem for the more complicated system of elastodynamics. In particular it is proven in [31] that from the HDN map associated to the equations 
of isotropic elastodynamics one can determine, under the assumption of simplicity of the metrics, the lengths of geodesics of the metrics defined by

$$
d s^{2}=c_{p}^{2}(x) e, \quad d s^{2}=c_{s}^{2}(x) e,
$$

where $c_{p}(x)=\sqrt{\frac{(\lambda+2 \mu)}{\rho}}, c_{s}(x)=\sqrt{\frac{\mu}{\rho}}$ denote the speed of compressional waves and shear waves respectively. Here $\lambda, \mu$ are the Lamé parameters and $\rho$ the density. The article by [10] in this book examines in detail the inverse problem of determining the metric from the associated boundary distance function, the so-called boundary rigidity problem in Riemannian geometry.

Using Mukhometov's result [27], [28] we can then recover both speeds from the HDN map. This shows in particular that if we know the density one can determine the Lamé parameters from the HDN map. By using the transport equation of geometrical optics, similar to (16), and the results on the ray transform (see for instance [34]), Rachele shows that under certain a-priori conditions one can also determine the density $\rho$ [32].

4. The scattering relation. In the presence of caustics (i.e. the exponential map is not a diffeomorphism) the expansion (6) is not valid since we cannot solve globally the Eikonal equation globally in $\Omega$. The solution of (4) is globally a Lagrangian distribution (see for instance [16]). These distributions can locally be written in the form

$$
u(t, x, \omega)=\int e^{i \phi(t, x, \omega, \theta)} a(t, x, \omega, \theta) d \theta
$$

where $\phi$ is a phase function and $a(t, x, \omega, \theta)$ is a classical symbol.

Every Lagrangian distribution is determined (up to smoother terms) by a Lagrangian manifold and its symbol. The Lagrangian manifold associated to $u(t, x, \omega)$ is the flow out from $t=x \cdot \omega, t<-R$ by the Hamilton vector field of $p_{g}=\tau^{2}-g^{-1}(x, \xi)$. Here $(\tau, \xi)$ are the dual variables to $(t, x)$ respectively. The projection in the $(x, \xi)$ variables of the flow is given by the flow out from $N$ by geodesic flow, that is the Lagrangian submanifold $L$ described above.

The scattering relation (also called lens map), $C_{g} \subset\left(T^{*}(\mathbf{R} \times \partial \Omega) \backslash\right.$ $0) \times\left(T^{*}(\mathbf{R} \times \partial \Omega) \backslash 0\right)$ of a metric $g=\left(g^{i j}\right)$ on $\bar{\Omega}$ with dual metric $g^{-1}=$ $\left(g_{i j}\right)$ is defined as follows. Consider bicharacteristic curves, $\gamma:[a, b] \rightarrow$ $T^{*}(\bar{\Omega} \times \mathbf{R})$, of the Hamilton function $p_{g}(t, x, \tau, \xi)=\tau^{2}-g^{-1}(x, \xi)$ which satisfy the following: $\gamma(] a, b[)$ lies in the interior, $\gamma$ intersects the boundary non-tangentially at $\gamma(a)$ and $\gamma(b)$, and time increases along $\gamma$. Then the canonical projection from $\left(T_{\mathbf{R} \times \partial \Omega}^{*}(\mathbf{R} \times \Omega) \backslash 0\right) \times\left(T_{\mathbf{R} \times \partial \Omega}^{*}(\mathbf{R} \times \Omega) \backslash 0\right)$ onto $\left.\left(T^{*}(\mathbf{R} \times \partial \Omega) \backslash 0\right) \times T^{*}(\mathbf{R} \times \partial \Omega) \backslash 0\right)$ maps the endpoint pair $(\gamma(b), \gamma(a))$ to a point in $C_{g}$. In other words $C_{g}$ gives the geodesic distance between points in the boundary and also the points and direction of exit of the geodesic if we know the point and direction of entrance. 
It is well-known that $C_{g}$ is a homogeneous canonical relation on $\left(T^{*}(\mathbf{R} \times \partial \Omega) \backslash 0\right) \times\left(T^{*}(\mathbf{R} \times \partial \Omega) \backslash 0\right)$. (See [14] for the concept of a scattering relation.) $C_{g}$ is, in fact, a diffeomorphism between open subsets of $T^{*}(\mathbf{R} \times \partial \Omega) \backslash 0$.

In analogy with Theorem 3.1 we have

THEOREM 4.1. Let $g_{i}, i=1,2$ be Riemannian metrics on $\bar{\Omega}$ satisfying the assumptions of Theorem 2.1. Then

$$
C_{g_{1}}=C_{g_{2}} .
$$

Sketch of Proof. Since by (5) we know the solutions of (4) outside $\Omega$. Therefore the associated Lagrangian manifolds to the Lagrangian distributions $u_{j}$ must be the same outside $\Omega$. By taking the projection of these Lagrangians onto the boundary we get the desired claim.

In the case that $(\Omega, g)$ is simple then the scattering relation doesn't give any new information. In fact $\left(\left(t_{1}, x_{1}, \tau, \xi_{1}\right),\left(t_{0}, x_{0}, \tau, \xi_{0}\right)\right) \in C_{g}$ if $t_{1}-$ $t_{0}=d_{g}\left(x_{1}, x_{0}\right)$ and $\xi_{j}=-\tau \frac{\partial d_{g}\left(x_{1}, x_{0}\right)}{\partial x_{j}}, j=0,1$. In other words $d_{g}$ is the generating function of the scattering relation.

This result was generalized in [15] to the case of the equations of elastodynamics with residual stress. It is shown that knowing the HDN map we can recover the scattering relations associated to $P$ and $S$ waves. For this one uses Lagrangian distributions with appropriate polarization.

The scattering relation contains all travel time data; not just information about minimizing geodesics as is the case of the boundary distance function. The natural conjecture is that this is enough to determine the metric up to isometry.

CONJECTURE 4.1. From the scattering relation we can determine the metric up to isometry.

We have developed in [36] an approach to this conjecture and derive the identity below. We will consider the case of subdomains of $\mathbf{R}^{3}$.

Let $x^{(0)} \in \Gamma, \xi^{(0)} \in S^{2}$ such that $\nu\left(x^{(0)}\right) \cdot g^{-1} \xi^{(0)}<0$. Here $g$ is either $g_{1}$ or $g_{2}$, while the initial conditions are the same for both metrics. We remark that if $\xi^{(0)} \cdot g^{-1} \xi^{(0)}=1$, then $s$ is the arc-length in (15).

Consider the Hamiltonian system (15) with the following initial conditions

$$
\left\{\begin{aligned}
\frac{d}{d s} x_{m} & =\sum_{j=1}^{n} g^{m j} \xi_{j}, \quad \frac{d}{d s} \xi_{m}=-\frac{1}{2} \sum_{i, j=1}^{n} \frac{\partial g^{i j}}{\partial x_{m}} \xi_{i} \xi_{j}, m=1, \ldots, n, \\
\left.x\right|_{s=-\rho} & =(-\rho, z),\left.\quad \xi\right|_{s=-\rho}=(1,0,0) .
\end{aligned}\right.
$$

Here $z \in \mathbf{R}^{2}, \rho>0$ is such that $g=e$ for $|x|>\rho$ and the solution $x=x(s, z), \xi=\xi(s, z)$ depends on the parameter $z$. If $g=e$, then $x=(s, z)=\left(s, z_{1}, z_{2}\right)$.

Denote the solution of (15) by $x=x\left(s, x^{(0)}, \xi^{(0)}\right), \xi=\xi\left(s, x^{(0)}, \xi^{(0)}\right)$. Let us introduce new notation

$$
X:=(x, \xi) .
$$


The solution to (15) related to $g_{1}$ and $g_{2}$, respectively, can therefore be written down as $X_{g_{j}}=X_{g_{j}}\left(s, X^{(0)}\right)=X_{g_{j}}\left(s, x^{(0)}, \xi^{(0)}\right)$.

Set $F(s):=X_{g_{2}}\left(t-s, X_{g_{1}}\left(s, X^{(0)}\right)\right)$. Here $t=t\left(X^{(0)}\right)$ is the length of the geodesics issued from $X^{(0)}$ with endpoint on $\Gamma$ and $t$ is independent of $g=g_{1}$ or $g=g_{2}$. We have $F(0)=X_{g_{2}}\left(t, X^{(0)}\right)=X_{g_{1}}\left(t, X^{(0)}\right)=F(t)$. Thus

$$
\int_{0}^{t} F^{\prime}(s) d s=0
$$

A calculation gives

$$
\begin{aligned}
F^{\prime}(s)= & -V_{g_{2}}\left(X_{g_{2}}\left(t-s, X_{g_{1}}\left(s, X^{(0)}\right)\right)\right) \\
& +\frac{\partial X_{g_{2}}}{\partial X^{(0)}}\left(t-s, X_{g_{1}}\left(s, X^{(0)}\right)\right) V_{g_{1}}\left(X_{g_{1}}\left(s, X^{(0)}\right)\right) .
\end{aligned}
$$

We claim that

$$
\begin{aligned}
& V_{g_{2}}\left(X_{g_{2}}\left(t-s, X_{g_{1}}\left(s, X^{(0)}\right)\right)\right) \\
& \quad=\frac{\partial X_{g_{2}}}{\partial X^{(0)}}\left(t-s, X_{g_{1}}\left(s, X^{(0)}\right)\right) V_{g_{2}}\left(X_{g_{1}}\left(s, X^{(0)}\right)\right) .
\end{aligned}
$$

Indeed, (24) follows from

$$
\begin{aligned}
0 & =\left.\frac{d}{d s}\right|_{s=0} X\left(T-s, X\left(s, X^{(0)}\right)\right) \\
& =-V\left(X\left(T, X^{(0)}\right)\right)+\frac{\partial X}{\partial X^{(0)}}\left(T, X^{(0)}\right) V\left(X^{(0)}\right), \quad \forall T
\end{aligned}
$$

after setting $T=t-s$. Therefore, (22), (23) and (24) combined together imply

$$
\int_{0}^{t} \frac{\partial X_{g_{2}}}{\partial X^{(0)}}\left(t-s, X_{g_{1}}\left(s, X^{(0)}\right)\right)\left(V_{g_{1}}-V_{g_{2}}\right)\left(X_{g_{1}}\left(s, X^{(0)}\right)\right) d s=0 .
$$

This identity is valid whenever the scattering relation of the two metrics is the same. Roughly speaking (25) is a non-linear integral equation on the difference of the metrics $\left(g_{1}\right)^{-1}$ and $\left(g_{2}\right)^{-1}$.

Formula (25) is the main result used in [36] to prove that if two metrics have the same lengths of geodesics and they are a-priori close to the Euclidean metric in an appropriate topology then they must be isometric. The idea is to linearize this identity at the Euclidean metric and use perturbation arguments to derive the result.

As mentioned in [34] and [25] this has been extended to a semiglobal result. Namely one of the metrics satisfies a curvature assumption and the other one is sufficiently close to the Euclidean metric. The result is that if the metrics have the same boundary distance function under these conditions then they must be isometric. 
5. The elliptic Dirichlet-to-Neumann map. In this section we consider the elliptic analog of the HDN map considered in Section 2. One of the motivations for this problem comes from Electrical Impedance Tomography (EIT). In this non-invasive inverse method one attempts to determine the electrical conductivity of a body by making voltage and current measurements at the boundary of the body. For anisotropic conductors the electrical properties of the medium depend on direction. Muscle tissue is a prime example of an anisotropic conductor. For instance for cardiac muscle tissue the transverse conductivity is 2.3 mho while the longitudinal one is 6.3 mho. An anisotropic conductivity is modeled as a positive definite symmetric metric $\gamma=\left(\gamma^{i j}\right)$. Under the assumption of no sources or sinks of currents the equation for the potential $u$, given a voltage potential $f$ on the boundary, is given by the solution of the Dirichlet problem

$$
\sum_{i, j=1}^{n} \frac{\partial}{\partial x_{i}}\left(\gamma^{i j} \frac{\partial u}{\partial x_{j}}\right)=0 \quad \text { on } \Omega ;\left.\quad u\right|_{\partial \Omega}=f .
$$

The DN map is defined by

$$
\Lambda_{\gamma}(f)=\sum_{i, j=1}^{n} \gamma^{i j} \frac{\partial u}{\partial x_{j}} \nu^{i}
$$

where $\nu^{i}$ denotes the $i^{\text {th }}$ component of $\nu$.

It was observed by Luc Tartar (see [22] for an account) that if $\psi: \bar{\Omega} \rightarrow$ $\bar{\Omega}$ is a diffeomorphism with $\left.\psi\right|_{\partial \Omega}=$ Identity then $\Lambda_{\widetilde{\gamma}}=\Lambda_{\gamma}$ where

$$
\widetilde{\gamma}=\left(\frac{(D \psi)^{T} \circ \gamma \circ(D \psi)}{|\operatorname{det} D \psi|}\right) \circ \psi^{-1}=: \psi_{*} \gamma .
$$

Here $D \psi$ denotes the (matrix) differential of $\psi,(D \psi)^{T}$ its transpose and the composition in (28) is to be interpreted as composition of matrices.

ConjeCture 5.1. Let $\gamma, \widetilde{\gamma}$ be conductivities satisfying $\Lambda_{\gamma}=\Lambda_{\tilde{\gamma}}$. Then there exists a diffeomorphism $\psi: \bar{\Omega} \longrightarrow \bar{\Omega}$, with $\left.\psi\right|_{\partial \Omega}=$ Identity so that $\widetilde{\gamma}=\psi_{*} \gamma$.

In dimensions $n \geq 3$ the problem can be reformulated as a geometric one. Given an anisotropic conductivity $\gamma=\left(\gamma^{i j}\right)$ we define $g^{i j}=$ $(\operatorname{det} \gamma)^{\frac{2}{n-2}} \gamma^{i j}$. The Riemannian metric is given by $\left(g_{i j}\right)=\left(g^{i j}\right)^{-1}$. The conductivity equation is replaced by the Laplace-Beltrami operator. We consider the solution of the elliptic boundary value problem

$$
\Delta_{g} u=0 \text { on } \Omega,\left.\quad u\right|_{\partial \Omega}=f .
$$

We define the EDN map by

$$
\Lambda_{g}(f)=\left.\sum_{i, j=1}^{n} \nu^{i} g^{i j} \frac{\partial u}{\partial x_{j}} \sqrt{\operatorname{det} g}\right|_{\partial \Omega}
$$


where $\left(\nu^{i}\right)=\nu$ is the outer unit normal to $\partial \Omega$. The inverse problem is to recover $g$ from $\Lambda_{g}$.

We note that under the correspondence between conductivities and Riemannian metrics indicated above the corresponding EDN maps satisfy $\Lambda_{g}=\Lambda_{\gamma}$. Similarly to (28) we have that for any diffeomorphism $\psi: \bar{\Omega} \longrightarrow$ $\bar{\Omega},\left.\quad \psi\right|_{\partial \Omega}=$ Identity

$$
\Lambda_{\psi^{*} g}=\Lambda_{g}
$$

where $\psi^{*} g$ denotes the pull-back of the metric $g$.

The problem can be formulated for arbitrary compact Riemannian manifolds with boundary. More specifically

CONJECTURE 5.2. Let $(M, g)$ be a compact Riemannian manifold with boundary of dimension $n \geq 3$. The pair $\left(\partial M, \Lambda_{g}\right)$ determines $(M, g)$ uniquely. Of course uniquely means up to an isometric copy.

The more general result proven about this conjecture is the following [24]

THEOREM $5.1(n \geq 3)$. Let $(M, g)$ be a real-analytic compact, connected Riemannian manifold with boundary. Let $\Gamma \subseteq \partial M$ be real-analytic and assume that $g$ is real-analytic up to $\Gamma$. Then $\left(\Lambda_{g}, \partial M\right)$ determines uniquely $(M, g)$.

This result is also valid if we measure the EDN map in an open subset of the boundary $\Gamma$ (measured for functions supported on that subset) under the a-priori assumption that $g$ is real-analytic in the interior and up to $\Gamma$.

Notice that Theorem 5.1 doesn't assume any condition on the topology of the manifold except for connectedness. An earlier result of [26] assumed that $(M, g)$ was strongly convex and simply connected and $\Gamma=\partial M$.

In the two dimensional case there is an additional obstruction. Namely the Laplace-Beltrami operator is conformally invariant. More precisely

$$
\Delta_{\alpha g}=\frac{1}{\alpha} \Delta_{g}
$$

for any function $\alpha, \alpha \neq 0$. Therefore we have that for $n=2$

$$
\Lambda_{\alpha\left(\psi^{*} g\right)}=\Lambda_{g}
$$

for any smooth function $\alpha \neq 0$ such that $\left.\alpha\right|_{\partial M}=1$.

Therefore the best we can recover by knowing $\Lambda_{g}$ is the conformal class of the metric $g$. That this is in fact the case was shown by Lassas and the author in [24]. We have:

TheOREM 5.2. Let $(M, g)$ be a compact Riemannian surface. Then the pair $\left(\partial M, \Lambda_{g}\right)$ determines uniquely the conformal class of $(M, g)$. Uniquely means up to an isometric copy.

Also this result only requires local measurements of the EDN map on an open subset of the boundary. 
A basic result which is used in all the results stated in this section is the following Lemma proved in [26]:

LEMMA 5.1. Let $(M, g)$ be a compact Riemannian manifold with boundary. Then $\Lambda_{g}$ determines the $C^{\infty}$-jet of the metric at the boundary in the following sense. If $g^{\prime}$ is another Riemannian metric on $M$ such that $\Lambda_{g}=\Lambda_{g^{\prime}}$, then there exists a diffeomorphism $\varphi: M \rightarrow M,\left.\varphi\right|_{\partial M}=$ Identity such that $g^{\prime}=\varphi^{*} g$ to infinite order at $\partial M$.

In other words Lemma 5.1 shows that the conjectures above are valid at the boundary. The proof of this result is done by showing that $\Lambda_{g}$ is a pseudodifferential operator of order 1 . Its full symbol, calculated in appropriate coordinates, determines the $C^{\infty}$-jet of the metric $g$ at the boundary.

In the two dimensional case it was proven in [38], under some regularity assumptions on the conductivity, that we can reduce the anisotropic case to the isotropic one. This uses fundamentally isothermal coordinates, which are not available in dimension $n \geq 3$. An anisotropic conductivity is a conductivity $\gamma^{i j}=\alpha(x) \delta^{i j}$ where the function $\alpha$ is a strictly positive function. The corresponding Riemannian metric in dimension $n \geq 3$ is then conformal to the Euclidean metric.

The following are the two basic results in the isotropic case:

THEOREM 5.3. Let $\Omega \subseteq \mathbf{R}^{2}$ be a bounded domain with smooth boundary. Let $\gamma^{(i)}, i=1,2$ be two isotropic conductivities in $C^{2}(\bar{\Omega})$. Then $\Lambda_{\gamma_{1}}=\Lambda_{\gamma_{2}}$ implies $\gamma_{1}=\gamma_{2}$.

TheOREM 5.4. Let $\Omega \subseteq \mathbf{R}^{n} n \geq 3$ be a bounded domain with smooth boundary. Let $\gamma^{(i)}, i=1,2$ be two $C^{2}$ isotropic Riemannian metrics. Then $\Lambda_{\gamma_{1}}=\Lambda_{\gamma_{2}}$ implies $\gamma_{1}=\gamma_{2}$.

The two dimensional case is due to Nachman [29] and the case of dimension three or higher to Sylvester and Uhlmann [40].

In the next two sections we outline the proof of these last two results.

5.1. Complex-geometrical optics solutions. In this section we outline the proof of Theorem 5.4. We actually prove a more recent result of Bukhgeim and the author [8] that states that is enough to measure the EDN map on particular subsets of the boundary. A crucial ingredient in the proof is the construction of complex geometrical optics (CGO) solutions of the Laplace-Beltrami operator when the Riemannian metric is isotropic. Another important element is the use of Carleman estimates.

The main result of CGO solutions is given by

LEMMA 5.2. Let $g$ be an isotropic Riemannian metric $g=\alpha e$ with $\alpha$ a scalar function satisfying $\alpha=1$ outside a large ball. Let $\rho \in \mathbf{C}^{n}, \rho \cdot \rho=0$. Then for $|\rho|$ sufficiently large, there exist solutions of $\Delta_{g} u=0$ of the form

$$
u=e^{\langle x, \rho\rangle} \alpha^{-\frac{1}{2}}\left(1+\psi_{g}(x, \rho)\right)
$$

with $\psi_{g} \underset{|\rho| \rightarrow \infty}{\longrightarrow} 0$ uniformly in compact sets. 
For more precise statements and a recent survey of other results using CGO see [45]. We remark that this method started with the pioneering work of Calderón [9].

One of the main difficulties in extending Theorem 5.4 to the general anisotropic case even in the case when $M$ is an open subset of Euclidean space is to construct an analog of (33) in this case.

The proof of Theorem 5.4 proceeds by proving a more general result. Namely one reduces the problem to consider the set of Cauchy data for solutions of the Schrödinger equation (see [45] for more details).

Let $n \geq 3$. Let $q \in L^{\infty}(\Omega)$. We define the set of Cauchy data for the associated Schrödinger equation by

$$
\mathcal{C}_{q}=\left\{\left(\left.u\right|_{\partial \Omega},\left.\frac{\partial u}{\partial \nu}\right|_{\partial \Omega}\right) \mid(\Delta-q) u=0 \quad \text { on } \Omega, u \in H^{1}(\Omega)\right\} .
$$

Theorem 5.5. Let $q_{i} \in L^{\infty}(\Omega), i=1,2$. Assume

$$
\mathcal{C}_{q_{1}}=\mathcal{C}_{q_{2}}
$$

Then $q_{1}=q_{2}$.

The proof of this result uses complex geometrical optics solutions of the Schrödinger equation. Let $q \in L^{\infty}\left(\mathbb{R}^{n}\right), n \geq 2$ have compact support. Then for $\rho \in \mathbb{C}^{n}, \rho \cdot \rho=0,|\rho|$ sufficiently large, one can construct solutions to

$$
(\Delta-q) u_{\rho}=0
$$

of the form

$$
u_{\rho}=e^{\langle x, \rho\rangle}\left(1+\psi_{q}(x, \rho)\right)
$$

with

$$
\left\|\psi_{q}(\cdot, \rho)\right\|_{H^{s}(\Omega)} \leq \frac{C}{|\rho|^{1-s}}, \quad 0 \leq s \leq 1,
$$

for some $C>0$ independent of $\rho$.

The function $\psi_{q}(x, \rho)$ solves

$$
\Delta_{\rho} \psi_{q}=q\left(1+\psi_{q}\right)
$$

where

$$
\Delta_{\rho} u=e^{-\langle x, \rho\rangle} \Delta\left(e^{\langle x, \rho\rangle} u\right) .
$$

The Schwartz kernel $G_{\rho}$ of $\Delta_{\rho}^{-1}$ is the so-called Faddeev's Green kernel [12]. The following estimate was proved in [41] $(n=2)$, [40] $(n \geq 3)$ for $-1<\delta<0$ and $\rho \in \mathbb{C}^{n}-0, \rho \cdot \rho=0$ :

$$
\left\|G_{\rho} f\right\|_{H_{\delta}^{s}} \leq C \frac{\|f\|_{L_{\delta+1}^{2}}}{|\rho|^{1-s}}
$$


Here $H_{\alpha}^{s}$ denotes the Sobolev space associated to the weighted $L^{2}$ space with norm given by

$$
\|f\|_{L_{\alpha}^{2}}^{2}=\int|f(x)|^{2}\left(1+|x|^{2}\right)^{\alpha} d x .
$$

A natural question is whether one can determine the potential by measuring the Cauchy data on strict subsets of the boundary. The only result known beyond the case of real-analytic potentials was proven in [8]. We describe the result below.

We first modify the set of Cauchy data to allow for more singular distributions on the boundary. We define the function space

$$
H_{\Delta}(\Omega)=\left\{u \in \mathcal{D}^{\prime}(\Omega) \mid u \in L^{2}(\Omega), \Delta u \in L^{2}(\Omega)\right\}
$$

$H_{\Delta}(\Omega)$ is a Hilbert space with the norm

$$
\|u\|_{H_{\Delta}(\Omega)}^{2}=\|u\|_{L^{2}(\Omega)}^{2}+\|\Delta u\|_{L^{2}(\Omega)}^{2} .
$$

For $u \in H_{\Delta}(\Omega)$, we have $\left.u\right|_{\partial \Omega} \in H^{-\frac{1}{2}}(\partial \Omega)$ and $\left.\frac{\partial u}{\partial \nu}\right|_{\partial \Omega} \in H^{-\frac{3}{2}}(\partial \Omega)$. We define the set of modified Cauchy data for $q \in L^{\infty}(\Omega)$ by

$$
\begin{aligned}
C_{q}=\{ & \left(\left.u\right|_{\partial \Omega},\left.\frac{\partial u}{\partial \nu}\right|_{\partial \Omega}\right) \in H^{-\frac{1}{2}}(\partial \Omega) \\
& \left.\times H^{-\frac{3}{2}}(\partial \Omega) \mid(\Delta-q) u=0 \text { in } \Omega, u \in H_{\Delta}(\Omega)\right\} .
\end{aligned}
$$

If 0 is not a Dirichlet eigenvalue of $\Delta-q$ in $\Omega$ then $C_{q}$ contains the graph of the Dirichlet-to-Neumann map $\Lambda_{q}$ conventionally defined on $H^{1 / 2}(\partial \Omega)$ by the relation $\Lambda_{q}(f)=\left.\frac{\partial u}{\partial \nu}\right|_{\partial \Omega}$, where $u \in H^{1}(\Omega)$ is a solution to the problem

$$
(\Delta-q) u=0 \quad \text { in } \Omega,\left.\quad u\right|_{\partial \Omega}=f
$$

i.e., $\left\{\left(f, \Lambda_{q}(f)\right) \mid f \in H^{1 / 2}(\partial \Omega)\right\} \subset C_{q}$.

Fix $\xi \in S^{n-1}=\left\{\xi \in \mathbf{R}^{n},|\xi|=1\right\}$. We define

$$
\partial \Omega_{+}(\xi)=\{x \in \partial \Omega \mid\langle\nu, \xi\rangle>0\}, \quad \partial \Omega_{-}(\xi)=\{x \in \partial \Omega \mid\langle\nu, \xi\rangle<0\}
$$

and for $\varepsilon>0$

(40) $\partial \Omega_{+, \varepsilon}(\xi)=\{x \in \partial \Omega \mid\langle\nu, \xi\rangle>\varepsilon\}, \quad \partial \Omega_{-, \varepsilon}(\xi)=\{x \in \partial \Omega \mid\langle\nu, \xi\rangle<\varepsilon\}$.

We also define the set of restricted Cauchy data

$$
C_{q, \varepsilon}=\left\{\left(\left.u\right|_{\partial \Omega},\left.\frac{\partial u}{\partial \nu}\right|_{\partial \Omega_{-, \varepsilon}(\xi)}\right) \mid(\Delta-q) u=0 \text { in } \Omega, u \in H_{\Delta}(\Omega)\right\} .
$$


The main result of [8] is

TheOrEm 5.6. Let $n \geq 3$ and $q_{i} \in L^{\infty}(\Omega), i=1,2$. Given $\xi \in S^{n-1}$ and $\varepsilon>0$, assume that $C_{q_{1}, \varepsilon}=C_{q_{2}, \varepsilon}$. Then $q_{1}=q_{2}$.

Theorem 5.6 has an immediate consequence in Electrical Impedance Tomography. We assume here now $\gamma$ is an isotropic conductivity, i.e, $\gamma_{i j}=$ $\gamma(x) \delta_{i j}$ with $\gamma \in C^{2}(\bar{\Omega})$ a strictly positive function on $\bar{\Omega}$.

As a direct consequence of Theorem 5.6 we prove

Corollary 5.1. Let $\gamma_{i} \in C^{2}(\bar{\Omega}), i=1,2$, be strictly positive. Given $\xi \in S^{n-1}$ and $\varepsilon>0$, assume that $\left.\gamma_{1}\right|_{\partial \Omega}=\left.\gamma_{2}\right|_{\partial \Omega}$ and

$$
\left.\Lambda_{\gamma_{1}}(f)\right|_{\partial \Omega_{-, \varepsilon}(\xi)}=\left.\Lambda_{\gamma_{2}}(f)\right|_{\partial \Omega_{-, \varepsilon}(\xi)} \quad \forall f \in H^{-\frac{1}{2}}(\partial \Omega) .
$$

Then $\gamma_{1}=\gamma_{2}$.

As far as we know, Theorem 5.6 (Corollary 5.1) is the first global uniqueness result for the Schrödinger equation (conductivity equation) in which the Cauchy data are given only on part of the boundary, beyond the case of a real-analytic potential.

A natural way to attack the problem of finding a potential from partial information of the Cauchy data is to construct solutions of the form (35) with $\psi_{q}=0$ on part of the boundary. As it is shown in [17] it is impossible in general to solve the Dirichlet problem for (37) with $\psi_{\rho}$ decaying (or even polynomially bounded in $\rho$.) In [8] it is shown that we can prescribe Dirichlet conditions for $\psi_{\rho}$ on particular subsets of the boundary. More precisely we have

LEMMA 5.3. Let $n \geq 2$. Let $\rho \in \mathbb{C}^{n}$ with $\langle\rho, \rho\rangle=0$ and $\rho=\tau(\xi+i \eta)$ with $\xi, \eta \in S^{n-1}$. Suppose that $f(\cdot, \rho /|\rho|) \in W^{2, \infty}(\Omega)$ satisfies $\partial_{\xi} f=\partial_{\eta} f=$ 0 , where $\partial_{\xi}$ denotes the directional derivative in the direction $\xi$. Then we can find solutions to $(\Delta-q) u=0$ in $\Omega$ of the form

$$
u(x, \rho)=e^{\langle x, \rho\rangle}\left(f\left(x, \frac{\rho}{|\rho|}\right)+\psi(x, \rho)\right),\left.\quad \psi\right|_{\partial \Omega_{-}(\xi)}=0
$$

with

$$
\|\psi(\cdot, \rho)\|_{L^{2}(\Omega)} \leq \frac{C}{\tau}, \quad \tau \geq \tau_{0}
$$

for some $C>0$ and $\tau_{0}>0$.

5.2. Carleman estimates. The proof of Theorem 5.6 and Lemma 5.3 uses Carleman estimates for the operator $\Delta_{\rho}$, which is not an elliptic operator if we consider the dependence on the parameter $\rho$, to construct the solutions and prove the main result. The use of a linear phase function in these Carleman estimates gives rise to the restriction on measuring the Cauchy data on particular subsets of the boundary. 
TheOREM 5.7. For $q \in L^{\infty}(\Omega)$ there exist $\tau_{0}>0$ and $C>0$ such that for all $u \in C^{2}(\bar{\Omega}),\left.u\right|_{\partial \Omega}=0$, and $\tau \geq \tau_{0}$ we have the estimate

$$
\begin{aligned}
& \tau^{2} \int_{\Omega}\left|e^{-\tau\langle x, \xi\rangle} u\right|^{2} d x+\tau \int_{\partial \Omega_{+}}\langle\xi, \nu\rangle\left|e^{-\tau\langle x, \xi\rangle} \partial_{\nu} u\right|^{2} d S \\
& \quad \leq C\left(\int_{\Omega}\left|e^{-\tau\langle x, \xi\rangle}(\Delta-q) u\right|^{2} d x-\tau \int_{\partial \Omega_{-}}\langle\xi, \nu\rangle\left|e^{-\tau\langle x, \xi\rangle} \partial_{\nu} u\right|^{2} d S\right) .
\end{aligned}
$$

Sketch of the Proof of Theorem 5.6. As before we let $\xi \in S^{n-1}$. Fix $k \in \mathbf{R}^{n}$ such that $\langle\xi, k\rangle=0$. Using Lemma 5.2 , we choose a solution $u_{2} \in H_{\Delta}(\Omega)$ to $\left(\Delta-q_{2}\right) u_{2}=0$ in $\Omega$ of the form

$$
u_{2}=e^{\left\langle x, \rho_{2}\right\rangle}\left(1+\psi_{q_{2}}\left(x, \rho_{2}\right)\right)
$$

with

$$
\rho_{2}=\tau \xi-i \frac{k+l}{2}
$$

where $\langle l, k\rangle=\langle l, \xi\rangle=0$ and $|k+l|^{2}=4 \tau^{2}$ (with these conditions $\left\langle\rho_{2}, \rho_{2}\right\rangle=$ $0)$. In dimension $n \geq 3$ we can always choose such a vector $l$. Since $C_{q_{1}, \varepsilon}=C_{q_{2}, \varepsilon}$, there is a solution $u_{1} \in H_{\Delta}(\Omega)$ to $\left(\Delta-q_{1}\right) u_{1}=0$ in $\Omega$ such that

$$
\left.u_{1}\right|_{\partial \Omega}=\left.u_{2}\right|_{\partial \Omega},\left.\quad \frac{\partial u_{1}}{\partial \nu}\right|_{\partial \Omega_{-, \varepsilon}(\xi)}=\left.\frac{\partial u_{2}}{\partial \nu}\right|_{\partial \Omega_{-, \varepsilon}(\xi)} .
$$

Let us denote $u:=u_{1}-u_{2}$ and $q:=q_{1}-q_{2}$. We have

$$
\left(\Delta-q_{1}\right) u=q u_{2} \quad \text { in } \Omega,\left.\quad u\right|_{\partial \Omega}=0 .
$$

Now it is easy to see that $\left.u\right|_{\partial \Omega}=0$ and $u \in H_{\Delta}(\Omega)$ implies that $u \in H^{2}(\Omega)$. Also Green's formula is valid for $v \in H_{\Delta}(\Omega)$. Thus we obtain

$$
\int_{\Omega}\left(\Delta-q_{1}\right) u \bar{v} d x=\int_{\Omega} q u_{2} \bar{v} d x=\int_{\Omega} u\left(\Delta-q_{1}\right) \bar{v} d x+\int_{\partial \Omega} \frac{\partial u}{\partial \nu} \bar{v} d S
$$

Now, we choose

$$
\bar{v}=e^{\left\langle x, \rho_{1}\right\rangle}\left(1+\psi_{q_{1}}\left(x, \rho_{1}\right)\right)
$$

as in $(35)$ to be a solution to $\left(\Delta-q_{1}\right) \bar{v}=0$, where

$$
\rho_{1}=-\tau \xi-i \frac{k-l}{2}
$$

with $\xi, k$, and $l$ as before so that $\left\langle\rho_{1}, \rho_{1}\right\rangle=0$. Notice that with this choice of $\rho_{j}, j=1,2$, we have

$$
\rho_{1}+\rho_{2}=-i k
$$


With these choices of $u_{2}$ and $v$, the identity (41) now reads

$$
\int_{\Omega} q u_{2} \bar{v}=\int_{\partial \Omega} \frac{\partial u}{\partial \nu} \bar{v} d S
$$

The final step in the proof is to show that the right hand side of (42) goes to 0 as $\tau \rightarrow \infty$.

By hypothesis,

$$
\left.\partial_{\nu} u\right|_{\partial \Omega_{-, \varepsilon}(\xi)}=0
$$

Then we have

$$
\int_{\partial \Omega} \frac{\partial u}{\partial \nu} \bar{v} d S=\int_{\partial \Omega \backslash \partial \Omega_{-, \varepsilon}} \frac{\partial u}{\partial \nu} \bar{v} d S=\int_{\partial \Omega_{+, \varepsilon}} \frac{\partial u}{\partial \nu} \bar{v} d S .
$$

The Cauchy-Schwarz inequality and the estimate (38) yields

$$
\begin{aligned}
\left|\int_{\partial \Omega} \frac{\partial u}{\partial \nu} \bar{v} d S\right| & =\left|\int_{\partial \Omega_{+, \varepsilon}} \frac{\partial u}{\partial \nu} e^{\left\langle x, \rho_{1}\right\rangle}\left(1+\psi_{q_{1}}\left(x, \rho_{1}\right)\right) d S\right| \\
& \leq \int_{\langle\xi, \nu\rangle \geq \varepsilon}\left|\frac{\partial u}{\partial \nu} e^{-\tau\langle\xi, x\rangle}\left(1+\psi_{q_{1}}\left(\cdot, \rho_{1}\right)\right)\right| d S \\
& \leq\left(\int_{\langle\xi, \nu\rangle \geq \varepsilon}\left|e^{-\tau\langle\xi, x\rangle} \partial_{\nu} u\right|^{2} d S\right)^{\frac{1}{2}}
\end{aligned}
$$

for some $C>0$. Now we use the Carleman estimate of Theorem 5.7 to obtain

(46) $\tau \varepsilon \int_{\partial \Omega_{+, \varepsilon}}\left|e^{-\tau\langle\xi, x\rangle} \partial_{\nu} u\right|^{2} d S \leq \tau \int_{\partial \Omega_{+}}\langle\xi, x\rangle\left|e^{-\tau\langle\xi, x\rangle} \partial_{\nu} u\right|^{2} d S$

$$
\begin{aligned}
& \leq \int_{\Omega}\left|e^{-\tau\langle\xi, x\rangle}\left(\Delta-q_{1}\right) u\right|^{2} d x=\int_{\Omega}\left|e^{-\tau\langle\xi, x\rangle} q u_{2}\right|^{2} d x \\
& \leq 2\left(\left\|q_{1}\right\|_{L^{\infty}(\Omega)}+\left\|q_{2}\right\|_{L^{\infty}(\Omega)}\right)^{2}\left(1+\left\|\psi_{2}\right\|_{L^{2}(\Omega)}^{2}\right) .
\end{aligned}
$$

Hence, we have proved that

$$
\left|\int_{\partial \Omega} \frac{\partial u}{\partial \nu} \bar{v} d S\right| \leq C \tau^{-1} \rightarrow 0, \quad \tau \rightarrow \infty .
$$

Now letting $\tau \rightarrow \infty$ gives

$$
\int_{\Omega} e^{-i\langle x, k\rangle} q(x) d x=0
$$

for all $k \perp \xi$. Changing $\xi \in S^{n-1}$ in a small conic neighborhood and using the fact that $\widehat{q}(k)$ is analytic we get that $q=0$ finishing the proof of Theorem 5.6. 
Sketch of Proof of Corollary 5.1. It is well known that we can reduce the problem to the case of the Schrödinger equation using the transformation $w=\gamma^{\frac{1}{2}} u$. If $u$ solves the conductivity equation $\operatorname{div}(\gamma \nabla u)=0$, then $w$ solves

$$
(\Delta-q) w=0 \quad \text { in } \Omega
$$

with $q=\frac{\Delta \sqrt{\gamma}}{\sqrt{\gamma}}$. It is easy to see that

$$
\Lambda_{q}(f)=\left.\gamma^{-\frac{1}{2}}\right|_{\partial \Omega} \Lambda_{\gamma}\left(\left.\gamma^{-\frac{1}{2}}\right|_{\partial \Omega} f\right)+\left.\frac{1}{2}\left(\gamma^{-1} \frac{\partial \gamma}{\partial \nu}\right)\right|_{\partial \Omega} f
$$

Now Kohn and Vogelius showed in [23] that given any open subset $\Gamma$ of $\partial \Omega$, if we know $\left.\Lambda_{\gamma}(f)\right|_{\Gamma}$ for all $f$ then we can determine $\left.\gamma\right|_{\Gamma}$ and $\left.\frac{\partial \gamma}{\partial \nu}\right|_{\Gamma}$, reducing therefore the proof of Corollary 5.1 to Theorem 5.6.

CONJECTURE 5.3. It is natural to expect that one needs to only measure the following subset of the Cauchy data to recover the potential. Let $\Gamma$ be an arbitrary open subset of the boundary. We define

$$
C_{q, \Gamma}=\left\{\left(\left.u\right|_{\partial \Omega},\left.\frac{\partial u}{\partial \nu}\right|_{\Gamma}\right) \mid(\Delta-q) u=0 \quad \text { in } \Omega, u \in H_{\Delta}(\Omega)\right\} .
$$

The conjecture is that if we know $C_{q, \Gamma}$ then we can recover the potential $q$. It would also be interesting to prove stability estimates and give a reconstruction of the potential under the conditions of Theorem 5.6.

6. The $\bar{\partial} \partial$ system. In this section we describe an extension of Nachman's result to $C^{1}(\bar{\Omega})$ conductivities due to Brown and the author [7]. Instead of reducing the conductivity equation to the Schrödinger equation we reduce it to a first order system (the $\bar{\partial} \partial$ system). We construct CGO solutions for this system. We combine this with the inverse scattering method developed for this system by Beals and Coifman [4] and L. Sung [37].

The main result of [7] is:

THEOREM 6.1. Let $n=2$. Let $\gamma \in C^{1}(\bar{\Omega}), \gamma$ strictly positive on $\bar{\Omega}$. Assume $\Lambda_{\gamma_{1}}=\Lambda_{\gamma_{2}}$. Then $\gamma_{1}=\gamma_{2}$ in $\Omega$.

Theorem 6.1 was extended to complex conductivities with small imaginary part in [13], using the $\bar{\partial} \partial$ method. Complex conductivities with small imaginary part arise naturally when considering Maxwell's equations for time harmonic waves with small frequency.

Logarithmic type stability estimates were proven in [3] for $C^{1+\epsilon}(\bar{\Omega})$ conductivities. A reconstruction method based on the uniqueness proof of [7] was developed by Knudsen and Tamasan [21] for $C^{1+\epsilon}(\bar{\Omega})$ conductivities.

As mentioned earlier, the proof of theorem 6.1 first reduces the conductivity equation to a first order system. We define

$$
q=-\frac{1}{2} \partial \log \gamma
$$


and a matrix potential $Q$ by

$$
Q=\left(\begin{array}{cc}
0 & q \\
\bar{q} & 0
\end{array}\right)
$$

Let also $D$ be the operator

$$
D=\left(\begin{array}{ll}
\bar{\partial} & 0 \\
0 & \partial
\end{array}\right)
$$

where $\bar{\partial}=\frac{1}{2}\left(\partial_{x_{1}}-i \partial_{x_{2}}\right)$.

An easy calculation shows that, if $u$ satisfies the conductivity equation $\operatorname{div}(\gamma \nabla u)=0$, then

$$
\left(\begin{array}{c}
v \\
w
\end{array}\right)=\gamma^{\frac{1}{2}}\left(\begin{array}{c}
\partial u \\
\bar{\partial} u
\end{array}\right)
$$

solves the system

$$
D\left(\begin{array}{c}
v \\
w
\end{array}\right)-Q\left(\begin{array}{c}
v \\
w
\end{array}\right)=0
$$

In [7] Brown and Uhlmann construct matrix solutions of (54) of the form

$$
\psi(z, k)=m(z, k)\left(\begin{array}{cc}
e^{i z k} & 0 \\
0 & e^{-i \bar{z} k}
\end{array}\right) .
$$

where $z=x_{1}+i x_{2}, k \in \mathbb{C}$ with $m \rightarrow 1$ as $|z| \rightarrow \infty$ in a sense to be described below. A simple calculation shows that $m$ from (55) satisfies in $\Omega$ the following equation

$$
D_{k} m-Q m=0,
$$

where $D_{k}$ is the operator

$$
D_{k}=\left(\begin{array}{cc}
(\bar{\partial}-i \bar{k}) & 0 \\
0 & (\partial+i k)
\end{array}\right)
$$

In order to explain the construction of $m$ we need a few more definitions. Let

$$
\Lambda_{k}(z)=\left(\begin{array}{cc}
e(z, k) & 0 \\
0 & e(z,-\bar{k})
\end{array}\right), \quad e(z, k)=e^{i(z k+\bar{z} \bar{k})}
$$

and for any matrix $A$, define the following operator

$$
E_{k} A=E_{k}\left(\begin{array}{ll}
a_{11} & a_{12} \\
a_{21} & a_{22}
\end{array}\right)=\left(\begin{array}{cc}
a_{11} & e(z,-\bar{k}) a_{12} \\
a_{21} e(z, k) & a_{22}
\end{array}\right) .
$$


Notice that

$$
D_{k}=E_{k}^{-1} D E_{k}
$$

Let $D^{-1}$ be the operator

$$
D^{-1}=\left(\begin{array}{cc}
\bar{\partial}^{-1} & 0 \\
0 & \partial^{-1}
\end{array}\right)
$$

where

$$
\bar{\partial}^{-1} f(z)=\frac{1}{2 \pi i} \int \frac{f(w)}{z-w} d w \wedge d \bar{w}
$$

and

$$
\partial^{-1} f(z)=\frac{1}{2 \pi i} \int \frac{f(w)}{\bar{z}-\bar{w}} d w \wedge d \bar{w} .
$$

We have from (57) that $D_{k}^{-1}=E_{k}^{-1} D^{-1} E_{k}$. We look for solutions of (56) among the solutions of the integral equation

$$
\left(I-D_{k}^{-1} Q\right) m(z)=I
$$

where $I$ is the $2 \times 2$ identity matrix. For a $2 \times 2$ matrix $A$, let $A^{d}$ and $A^{\text {off }}$ denote its diagonal respectively off-diagonal part. If

$$
J=\frac{1}{2}\left(\begin{array}{cc}
-i & 0 \\
0 & i
\end{array}\right)
$$

we define the operator $\mathcal{J}$ by

$$
\mathcal{J} A=[J, A]=2 J A^{\text {off }}=-2 A^{\text {off }} J .
$$

To end with the preliminary notation, we recall the definition of the weighted $L^{p}$ space

$$
L_{\alpha}^{p}\left(\mathbb{R}^{2}\right)=\left\{f ; \int\left(1+|x|^{2}\right)^{\alpha}|f(x)|^{p} d x<\infty\right\} .
$$

The next result gives the solvability of (56) in an appropriate space.

TheOREM 6.2. Let $Q \in L^{\infty}\left(\mathbb{R}^{2}\right)$ and compactly supported. Assume that $Q$ is a hermitian matrix. Choose $r$ so that $r<2$ and then $\beta$ so that $\beta r>2$. Then the operator $\left(I-D_{k}^{-1} Q\right)$ is invertible in $L_{-\beta}^{r}$. Moreover the inverse is differentiable in $k$ in the strong operator topology.

Theorem 6.2 implies the existence of solutions of the form (55) with $m-1 \in L_{-\beta}^{r}\left(\mathbb{R}^{2}\right)$.

We remark that the proof of Theorem 6.2 consists in showing that the integral equation (58) is of Fredholm type in $L_{-\beta}^{r}$. The fact that it has 
been a trivial kernel follows by showing that if $\left(I-D_{k}^{-1} Q\right) n(z, k)=0$, then $n \in L^{p}$, for all $p>2$, satisfies a pseudoanalytic equation in the $z$ variable. By the standard Liouville's theorem for pseudoanalytic equations with coefficients in $L^{p}, p>2$ it follows that $n=0$.

Next we compute $\frac{\partial}{\partial \bar{k}} m(z, k)$.

TheOREM 6.3. Let $m$ be the solution of (56) with $m-1 \in L_{-\beta}^{r}\left(\mathbb{R}^{2}\right)$. Then

$$
\frac{\partial}{\partial \bar{k}} m(z, k)-m(z, \bar{k}) \Lambda_{k}(z) S_{Q}(k)=0
$$

where the scattering data $S_{Q}$ is given by [4]

$$
S_{Q}(k)=-\frac{1}{\pi} \mathcal{J} \int_{\mathbb{R}^{2}} E_{k} Q m d \mu
$$

where $d \mu$ denotes Lebesgue measure in $\mathbb{R}^{2}$.

A further calculation shows that

$$
S_{Q}(k)=\frac{i}{\pi} \int_{\mathbb{R}^{2}}\left(\begin{array}{cc}
0 & e(z,-\bar{k}) q(z) m_{22}(z, k) \\
-e(z, k) \bar{q}(z) m_{11}(z, k) & 0
\end{array}\right) d \mu .
$$

The behavior of $m$ in the $k$ variable is given by the following result:

TheOREM 6.4. Let $Q \in L^{\infty}\left(\mathbb{R}^{2}\right)$ and compactly supported. Then there exists $R=R(Q)$ so that for all $q>2$

$$
\sup _{z}\|m(z, \cdot)-1\|_{L^{q}\{k ;|k|>R\}} \leq C\|Q\|_{L^{\infty}}^{2}
$$

where the constants depend on $q$ and the diameter of the support of $Q$. Outline of proof of Theorem 6.1 We know [2], [23], [30], [42] that if $\gamma_{i} \in$ $C^{1}(\bar{\Omega})$ and $\Lambda_{\gamma_{1}}=\Lambda_{\gamma_{2}}$, then $\left.\partial^{\alpha} \gamma_{1}\right|_{\partial \Omega}=\left.\partial^{\alpha} \gamma_{2}\right|_{\partial \Omega} \forall|\alpha| \leq 1$. Therefore we can extend $\gamma_{i} \in C^{1}\left(\mathbb{R}^{2}\right), \gamma_{1}=\gamma_{2}$ in $\mathbb{R}^{2} \backslash \Omega$ and $\gamma_{i}=1$ outside a large ball. Thus $Q_{i} \in L^{\infty}\left(\mathbb{R}^{2}\right), i=1,2$. The proof follows the following steps.

Step 1. $\Lambda_{\gamma_{1}}=\Lambda_{\gamma_{2}} \Rightarrow S_{Q_{1}}=S_{Q_{2}}=: S$. With these extensions, we observe that for each $j$ the scattering data $S_{Q_{j}}(k), j=1,2$, has the representation

$$
\begin{aligned}
S_{Q_{j}}(k)=-2 J & \int_{\mathbf{R}^{2}}\left(\begin{array}{cc}
0 & \bar{\partial} \psi_{j}^{12} e^{-i z \bar{k}} \\
\partial \psi_{j}^{21} e^{i \bar{z} k} & 0
\end{array}\right) d \mu(z) \\
=-2 J & {\left[\int_{\mathbf{R}^{2} \backslash \Omega}\left(\begin{array}{cc}
0 & \bar{\partial} \psi_{j}^{12} e^{-i z \bar{k}} \\
\partial \psi_{j}^{21} e^{i \bar{z} \bar{k}} & 0
\end{array}\right) d \mu(z)\right.} \\
& \left.+\int_{\partial \Omega}\left(\begin{array}{cc}
0 & \bar{\nu} \psi_{j}^{12} e^{-i z \bar{k}} \\
\nu \psi_{j}^{21} e^{i \bar{z} \bar{k}} & 0
\end{array}\right) d \mu(z)\right] .
\end{aligned}
$$

The formula for $S_{Q_{j}}$ uses the complexified normal to the boundary

$$
\nu(z)=\nu_{1}(z)+i \nu_{2}(z), \bar{\nu}(z)=\nu_{1}(z)-i \nu_{2}(z)
$$


with $\left(\nu_{1}(z), \nu_{2}(z)\right)$ the unit outer normal at $z \in \partial \Omega$.

From this expression for $S_{Q_{j}}, j=1,2$, we see that if we can show

$$
\psi_{1}(z, k)=\psi_{2}(z, k) \quad \text { in } \mathbf{R}^{2} \backslash \bar{\Omega},
$$

then $S_{Q_{1}}=S_{Q_{2}}$.

The last formula follows by using a very similar argument to Lemma 2.6 in [41].

Step 2. Let $\widetilde{m}=m_{1}-m_{2}$. Using the $\bar{\partial}$-equation (60) and Step 1 we conclude that

$$
\frac{\partial}{\partial \bar{k}} \widetilde{m}(z, k)-\widetilde{m}(z, \bar{k}) \Lambda_{k}(z) S(k)=0
$$

With the elements of $\widetilde{m}$ we form the following four functions

$$
\begin{aligned}
& \left.u_{ \pm}(k, z)\right)=\widetilde{m}_{11}(z, k) \pm \overline{\widetilde{m}_{12}(z, \bar{k})} \\
& \left.v_{ \pm}(k, z)\right)=\widetilde{m}_{21}(z, k) \pm \overline{\widetilde{m}_{22}(z, \bar{k})}
\end{aligned}
$$

each of which lies in $L^{q}\left(\mathbb{R}^{2}\right)$ in the $k$-variable and satisfies, for a fixed $z$, a pseudoanalytic equation in the $k$-variable,

$$
\frac{\partial}{\partial \bar{k}} w(z, k)=r(z, k) \overline{w(z, k)} \text {. }
$$

where $r(z, k)$ is some component of $S$ multiplied by a complex coefficient of norm 1.

Step 3. In [7] it was shown that, for $Q \in L_{c}^{\infty}$ with $Q^{*}=Q$, we have that

$$
\int \operatorname{tr} S_{Q} S_{Q}^{*} \leq \int \operatorname{tr} Q Q^{*}
$$

This shows that $S_{Q} \in L^{2}$. Consequently, for each fixed $z$ we have the map $k \rightarrow r(z, k)$ is in $L^{2}\left(\mathbb{R}^{2}\right)$.

Step 4. Prove that $u_{ \pm}=v_{ \pm}=0$, hence $\widetilde{m}=0$ or $m_{1}=m_{2}$. Then it is easy to show $Q_{1}=Q_{2}$ and therefore $\gamma_{1}=\gamma_{2}$.

To do this we need the following generalization of Liouville Theorem for pseudoanalytic functions proven in [7].

Lemma 6.1. Let $f \in L^{2}\left(\mathbb{R}^{2}\right)$ and $w \in L^{p}\left(\mathbb{R}^{2}\right)$ for some finite $p$. Assume that $w e^{\bar{\partial}^{-1} f}$ is analytic. Then $w=0$. Let us define

$$
\begin{aligned}
& \widetilde{u}_{ \pm}=u_{ \pm} e^{\bar{\partial}^{-1} r} \\
& \widetilde{v}_{ \pm}=v_{ \pm} e^{\bar{\partial}^{-1} r} .
\end{aligned}
$$

It is easy to check that $\widetilde{u}_{ \pm}$and $\widetilde{v}_{ \pm}$are analytic. By the lemma above we conclude that $u_{ \pm}=v_{ \pm}=0$ which in turn gives $m_{1}=m_{2}$. It is easy to show $Q_{1}=Q_{2}$ and therefore $\gamma_{1}=\gamma_{2}$, concluding the proof of the Theorem 6.1. 
The idea of the proof of Lemma 6.1 is the observation that since $r \in$ $L^{2}\left(\mathbb{R}^{2}\right), f=\bar{\partial}^{-1} r$ is in $V M O\left(\mathbb{R}^{2}\right)$ (the space of functions with vanishing mean oscillation) and thus is $O(\log |z|)$ as $|z| \rightarrow \infty$. Hence $e^{f} w \in L^{\widetilde{p}}$ for $\tilde{p}>p$. By Liouville's theorem it follows that $e^{f} w=0$. The details can be found in $[7]$.

7. Final remarks. It was shown in Section 2 that from the hyperbolic Dirichlet- to-Neumann map we can recover the boundary distance function, assuming that the domain is geodesically convex. Is there any connection between the elliptic Dirichlet to Neumann map and the boundary distance function $d_{g}$ ? To know the EDN map is the same as knowing the set of Cauchy data

$$
C_{g}=\left\{\left(\left.u\right|_{\partial \Omega}, \Lambda_{g}\left(\left.u\right|_{\partial \Omega}\right)\right)\right\}
$$

This set is resemblant of the scattering relation defined in Section 4. The scattering relation is a Lagrangian manifold in finite dimensions and the set of Cauchy data a Lagrangian manifold in infinite dimensions. It would be very interesting to find a more direct relationship between these two sets without, of course, constructing the metric first.

\section{REFERENCES}

[1] G. Alessandrini, Stable determination of conductivity by boundary measurements, App. Anal. 27 (1988), 153-172.

[2] G. Alessandrini, Singular solutions of elliptic equations and the determination of conductivity by boundary measurements, J. Diff. Equations 84 (1990), $252-272$.

[3] J. A. BARCeló, T. BARceló, AND A. Ruiz, Stability of the inverse conductivity problem in the plane for less regular conductivities, J. Diff. Equations 173 (2001), no. 2, 231-270.

[4] R. Beals and R. Coifman, The spectral problem for the Davey-Stewarson and Ishimori hierarchies, in Nonlinear evolution equations: Integrability and spectral methods, Manchester University Press (1988), 15-23.

[5] M. Belishev, Boundary control in reconstruction of manifolds and metrics (the $B C$ method), Inverse Problems 13 (1997), no. 5, R1-R45.

[6] M. Belishev And Y. Kurylev, To the reconstruction of a Riemannian manifold via its spectral data (BC-method), Comm. PDE 17 (1992), 767-804.

[7] R. Brown And G. Uhlmann, Uniqueness in the inverse conductivity problem for nonsmooth conductivities in two dimensions, Comm. Partial Diff. Equations 22 (1997), 1009-1027.

[8] A. Bukhgeim and G. Uhlmann, Determining a potential from partial Cauchy data, Comm. PDE 27 (2002), 653-668.

[9] A.P. Calderón, On an inverse boundary value problem, Seinar on Numerical Analysis and its Applications to Continuum Physics, Soc. Brasileira de Matemática, Río de Janeiro (1980), 65-73.

[10] C. Croke, This IMA Volume in Mathematics and its Applications, Geometric methods in inverse problems and PDE control, Editors: Christopher B. Croke, Irena Lasiecka, Gunther Uhlmann, and Michael Vogelius. Publisher: SpringerVerlag, New York.

[11] J.J. DuistermaAt, Fourier Integral Operators, Birkhäuser. 
[12] L. FADDEev, Growing solutions of the Schrödinger equation, Dokl. Akad. Nauk SSSR 165 (1965), 514-517 (translation in Sov. Phys. Dokl. 10, 1033).

[13] E. FRANCINI, Recovering a complex coefficient in a planar domain from the Dirichlet -to-Neumann map, Inverse Problems 16 (2000), 107-119.

[14] V. Guillemin, Sojourn times and asymptotic properties of the scattering matrix, Proceedings of the Oji Seminar on Algebraic Analysis and the RIMS Symposium on Algebraic Analysis (Kyoto Univ., Kyoto, 1976), Publ. Res. Inst. Math. Sci. 12 (1976/77), supplement, 69-88.

[15] S. Hansen AND G. Uhlmann, Propagation of polarization for the equations in elastodynamics with residual stress and travel times, to appear Math. Annalen.

[16] L. Hörmander, The Analysis of Linear Partial Differential Operators, Vol III, Springer-Verlag Berlin Heidelberg New York Tokyo (1985).

[17] M. Ikenata, The enclosure method and its applications, International Society for Analysis, Applications and Computation, Vol. 9, Kluwer Academic Pub., DORDRECHT (2001), 87-203 (edited by S. Saito, N. Hayashi, and M. Yamamoto.)

[18] A. Katchalov, Y. Kurylev, and M. Lassas, Inverse boundary spectral problems, Chapman\&Hall/CRC, 2001.

[19] A. Katchalov, Y. Kurylev, and M. Lassas, This IMA Volume in Mathematics and its Applications, Geometric methods in inverse problems and PDE control, Editors: Christopher B. Croke, Irena Lasiecka, Gunther Uhlmann, and Michael Vogelius. Publisher: Springer-Verlag, New York.

[20] A. Katsuda, Y. KuRYLEv, AND M. Lassas, Stability on inverse boundary spectral problem, in: New Geom. and Anal. Meth. in Inv. Probl. (Eds. Y. Kurylev and E. Somersalo), Springer Lect. Notes, to appear.

[21] K.Knudsen and A. Tamasan, Reconstruction of less regular conductivities in the plane, MSRI preprint series, Berkeley, 2001.

[22] R. Kohn And M. Vogelius, Identification of an unknown conductivity by means of measurements at the boundary, in Inverse Problems, edited by D. McLaughlin, SIAM-AMS Proc. no. 14, Amer. Math. Soc, Providence (1984), 113-123.

[23] R. Kohn And M. Vogelius, Determining conductivity by boundary measurements, Comm. Pure Appl. Math. 37 (1984), 289-298.

[24] M. Lassas and G. Uhlmann, On determining a Riemannian manifold from the Dirichlet-to-Neumann map, Annales Scientifiques de l' Ecole Normale Superiéure 34 (2001), 771-787.

[25] M. Lassas, V. Sharafutdinov, and G. Uhlmann, Semiglobal boundary rigidity for Riemannian metrics, to appear Math. Annalen.

[26] J. Lee And G. Uhlmann, Determining anisotropic real-analytic conductivities by boundary measurements, Comm. Pure Appl. Math. 42 (1989), 1097-1112.

[27] R.G. Muknometov, A problem of reconstructing a Riemannian metric, Siberian Math. J. 22 (1982), 420-433.

[28] R.G. Muknometov, The reconstruction problem of a two-dimensional Riemannian metric, and integral geometry (Russian), Dokl. Akad. Nauk SSSR 232 (1977), no. 1, 32-35

[29] A. Nachman, Global uniqueness for a two-dimensional inverse boundary value problem, Ann. of Math. (2) 143 (1996), 71-96.

[30] A. Nachman, Reconstructions from boundary measurements, Annals of Math. 128, (1988), 531-587.

[31] L. RAChele, An inverse problem in elastodynamics: Determination of the wave speeds in the interior, J. Diff Eqs. 162 (2000), 300-325.

[32] L. RAChELE, Uniqueness of the density in an inverse problem for isotropic elastodynamics, to appear Trans. AMS.

[33] L. Robianno And Zuilly, Uniqueness in the Cauchy problem for operators with partially holomorphic coefficients, Invent. Math. 131 (1998), 493-539. 
[34] V.A. Sharafutdinov, This IMA Volume in Mathematics and its Applications, Geometric methods in inverse problems and PDE control, Editors: Christopher B. Croke, Irena Lasiecka, Gunther Uhlmann, and Michael Vogelius. Publisher: Springer-Verlag, New York.

[35] P. Stefanov and G. Uhlmann, Stability estimates for the hyperbolic Dirichlet to Neumann map in anisotropic media, J. Funct. Anal. 154 (1998), 330-358.

[36] P. Stefanov and G. Uhlmann, Rigidity for metrics with the same lengths of geodesics, Math. Res. Lett. 5 (1998), 83-96.

37] L. Sung, An inverse scattering transform for the Davey-Stewartson II equations. I, II, III, J. Math. Anal. Appl. 183 (1994), 121-154, J. Math. Anal. Appl. 183 (1994), 289-325. J. Math. Anal. Appl. 183 (1994), 477-494.

[38] J. Sylvester, An anisotropic inverse boundary value problem, Comm. Pure Appl. Math. (1990), 201-232.

[39] J. Sylvester and G. Uhlmann, Inverse problems in anisotropic media, Contemp. Math. 122 (1991), 105-117.

[40] J. Sylvester and G. Uhlmann, A global uniqueness theorem for an inverse boundary value problem, Ann. of Math. 125 (1987), no. 2, 153-169.

[41] J. Sylvester and G. Uhlmann, A uniqueness theorem for an inverse boundary value problem in electrical prospection, Comm. Pure Appl. Math. 39 (1986), 91-112

[42] J. Sylvester and G. Uhlmann, Inverse boundary value problems at the boundary - continuous dependence, Comm. Pure Appl. Math. 41 (1988), 197-221.

[43] D. TATARU, Unique continuation for solutions to PDE's; between Hörmander's theorem and Holmgren's theorem, Comm. Partial Differential Equations 20 (1995), 855-884.

[44] D. Tataru. This IMA Volume in Mathematics and its Applications, Geometric methods in inverse problems and PDE control, Editors: Christopher B. Croke, Irena Lasiecka, Gunther Uhlmann, and Michael Vogelius. Publisher: SpringerVerlag, New York.

[45] G. Uhlmann, Developments in inverse problems since Calderón's foundational paper, chapter 19 of Essays in Harmonic Analysis and Partial Differential Equations, University of Chicago Press, edited by M. Christ, C. Kenig, C. Sadosky (1999) 